\title{
Precursor-Product Relationship between Pools of Very Low Density Lipoprotein Triglyceride
}

\author{
Philip J. Barter and Paul J. Nestel \\ From the Department of Clinical Science, The John Curtin School of Medical \\ Research, the Australian National University, Canberra, Australia
}

A B S T RAC T The process of removal of triglyceride from the plasma may involve a sequential conversion of larger to smaller glyceride-rich lipoproteins. This has been studied within the species of lipoproteins comprising the very low density lipoproteins (VLDL) which transport the bulk of endogenously formed triglyceride. Palmitic acid- ${ }^{14} \mathrm{C}$ which was used to label the plasma glycerides was administered either as a prolonged constant infusion or as a pulse label. The specific activitytime curves of triglyceride fatty acids (TGFA) were analyzed both in total VLDL and in two subfractions of VLDL. The nature of the curves for total VLDL that were observed during the constant infusions were consistent with slow isotopic equilibration of precursors of VLDL-TGFA or with the presence of a precursor-product relationship between different components of VLDL-TGFA. The curves did not indicate any detectable differences in (fractional) turnover rates of independently metabolized pools of VLDLTGFA. Differences in the specific activity-time curves of TGFA in two subfractions of VLDL ( $\mathrm{Sf}>100$ and Sf 20-100) were consistent with a precursor-product relationship between TGFA in the two subfractions; again there was no indication of significant differences in (fractional) turnover rates. The specific activity-time curves of TGFA in the two subfractions of VLDL that were obtained with single injections of radio-palmitate showed a consistent difference in the rates at which TGFA became labeled in the two subfractions, being slower in the Sf $20-100$ fraction. The findings from all experiments when considered together, were compatible with a precursor-product relationship that suggested that larger VLDL were converted to progressively smaller species as triglyceride was being removed.

Received for publication 11 May 1971 and in revised form 15 July 1971.

\section{INTRODUCTION}

Triglyceride that is newly synthesized in the liver is secreted into the plasma predominantly in the very low density lipoproteins (VLDL) ${ }^{1}(1)$. The VLDL comprise a wide morphological spectrum with the lipid to protein ratio increasing with size $(2-4)$. It is not clear whether all the triglyceride transported in VLDL represents a kinetically homogeneous compartment. A recent report from our laboratory showed consistent differences in the specific activity of triglyceride fatty acid (TGFA) transported in VLDL of different sizes during a constant infusion of palmitic acid ${ }^{14} \mathrm{C}(5)$. We suggested that larger VLDL might be degraded to smaller one during the process of triglyceride removal, although differences in the (fractional) turnover rates ${ }^{2}$ of TGFA in the various subclasses of VLDL could not be excluded.

In the present studies, the specific activity of TGFA in total VLDL and in two subfractions of VLDL has been measured during prolonged constant infusions and after single injections of palmitic acid ${ }^{14} \mathrm{C}$. We have attempted to determine whether the observed differences between the subfractions are the result of differing (fractional) turnover rates of independently metabolized pools or whether they indicate that TGFA transported in smaller VLDL is initially secreted into the plasma in larger lipoproteins. Our findings support the latter suggestion.

\section{METHODS}

\section{Experimental procedures}

15 subjects were studied. There were eight male and one female normal volunteers ${ }^{3}$ aged 19-42, two males aged 43

\footnotetext{
${ }^{1}$ Abbreviations used in this paper: TGFA, triglyceride fatty acids; VLDL, very low density lipoproteins.

2 (Fractional) turnover rate $=$ fraction of pool turning over per unit time.

${ }^{3}$ The nature of the studies was fully explained to all subjects and signed constant was obtained from the normal volunteers.
} 
and 52 with hepatic cirrhosis (4 and 11), two males aged 39 and 43 ( 1 and 13), and two females aged 58 and 61 (2 and 12) with type IV hyperlipoproteinemia (6). All subjects had been eating their habitual diets and studies were commenced in the morning after an overnight fast. Two kinds of study were carried out in which palmitic acid $-1-{ }^{14} \mathrm{C}$ was infused either at a constant rate or as a pulse injection.

Constant infusions. A venous catheter was inserted in each arm and kept patent by a slow infusion of isotonic saline. $45 \mathrm{~min}$ later an infusion of palmitic acid-1 $-{ }^{14} \mathrm{C}$ (The Radiochemical Centre, Amersham, England) complexed as the sodium salt to human albumin (7) was commenced via one catheter. The infusion was continued at a rate of 0.2 $\mathrm{ml} / \mathrm{min}$ (using a Harvard constant infusion pump Harvard Apparatus Co., Millis, Mass.) for 7-15 hr. A total of 25-75 $\mu \mathrm{Ci}$ was given to each subject. $10 \mathrm{ml}$ of blood was collected into chilled tubes containing $10 \mathrm{mg}$ of dipotassium EDTA from the catheter in the opposite arm at 30-60 min intervals after the first $1 \frac{1}{2} \mathrm{hr}$ of palmitate infusion. Plasma was separated at $4^{\circ} \mathrm{C}$ within $1 \mathrm{hr}$ of blood collection.

Single injections. A single injection of palmitic acid $-1-{ }^{14} \mathrm{C}$ was administered intravenously and blood was collected from an indwelling catheter in the opposite forearm at 15-30 min intervals for $4 \frac{1}{2}-6 \mathrm{hr}$.

\section{Analytical procedures}

The VLDL were separated from portions of plasma by preparative ultracentrifugation (8). In seven studies, two subclasses of VLDL ( $\mathrm{Sf}>100$ and Sf 20-100) were isolated from $3 \mathrm{ml}$ portions of plasma by the method described by Gustafson, Alaupovic, and Tierman (4). No further attempt was made to purify these subclasses by repeated ultracentrifugation since the aim was simply to obtain two fractions of VLDL containing predominantly larger or smaller lipoproteins.

The total VLDL and the subclasses were extracted in the solution described by Dole (9). Portions of the lipid extract were assayed for triglyceride content (10) and radioactivity. The latter was determined with an efficiency of $80 \%$ in a liquid scintillation system using $0.3 \%$ PPO in toluene as scintillator. Separation of the VLDL lipids by thin-layer silicic acid chromatography (hexane-ethermethanol-acetic acid, $180: 40: 6: 4$, solvent) had shown that $>96 \%$ of the radioactivity was in the triglyceride fraction. There was less than $7 \%$ variation in triglyceride concentration during any experiment.

Free fatty acid (FFA) specific radioactivity was measured in whole plasma that had been extracted in Dole's solution (9). Acidic and neutral lipids were separated according to Borgström (11) with a recovery of $96 \%$. During constant infusions of radiopalmitate the "steady-state" FFA specific activity varied by less than $15 \%$.

\section{THEORETICAL CONSIDERATIONS}

Previous experiments had shown consistent differences in the specific activity of TGFA transported in VLDL of different sizes during a $2 \frac{1}{2} \mathrm{hr}$ constant infusion of palmitic acid- ${ }^{14} \mathrm{C}(5)$. The findings were consistent with either of two possibilities (or a combination of the two possibilities). (a) That TGFA in VLDL of different sizes exist as independently metabolized pools in which the (fractional) turnover rate of TGFA varies as a function of VLDL particle size, or (b) that TGFA trans- ported in smaller VLDL were initially secreted into the plasma in larger lipoproteins. The present experiments attempt to differentiate between the two propositions.

Plasma FFA are a major precursor of VLDL-TGFA in the fasting state (1). When tracer amounts of labeled FFA are infused at a constant rate isotopic equilibration of intermediates between plasma FFA and VLDLTGFA occurs sequentially until ultimately the VLDLTGFA attain a constant specific radioactivity, C, equivalent to that of the immediate precursor. Hence the equilibrated specific activity of the immediate precursor can be deduced without the necessity of defining a precise compartment.

If during a constant infusion of labeled precursor it is assumed that the specific activity of the immediate precursor of VLDL-TGFA is constant at level $\mathrm{C}$ from zero time, and if the TGFA in VLDL is assumed to represent a single pool, then (reference 12)

$$
\mathrm{S}_{\mathrm{TG}}=\mathrm{C}\left(1-\mathrm{e}^{-\mathrm{kt}}\right)
$$

where $\mathrm{k}$ is the (fractional) turnover rate of VLDLTGFA and $S_{T G}$ denotes the VLDL-TGFA specific activity. Converting equation 1 to logarithms,

$$
-\log \left(1-\frac{\mathrm{S}_{\mathrm{TG}}}{\mathrm{C}}\right)=\mathrm{kt}
$$

so that the plot of $-\log \left(1-\left[\mathrm{S}_{\mathrm{TG}} / \mathrm{C}\right]\right)$ against time will be linear with a gradient equal to $\mathrm{k}$ (Fig. 1a).

If TGFA in VLDL exist as independently metabolized pools possessing differing (fractional) turnover rates, the plot will not be linear, but rather will possess a gradient decreasing with time; as faster pools reach isotopic equilibrium the curve will reflect the (fractional) turnover rates of the slower pools (Fig. 1b).

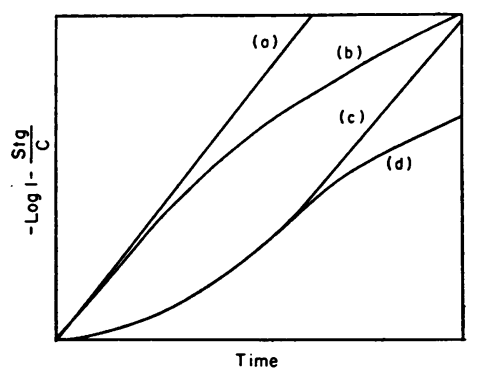

FIGURE 1 Theoretical curves of $-\log \left(1-\left[\mathrm{S}_{\mathrm{Ta}} / \mathrm{C}\right]\right)$ (see text) plotted against time during the constant infusion of a labeled precursor. (a) A homogeneous VLDL triglyceride pool with instantaneous isotopic equilibration of all precursors. (b) More than one kinetically distinct pool (differing turnover rates) of VLDL triglyceride with instantaneous isotopic equilibration of all precursors. (c) A homogeneous VLDL triglyceride pool with precursors equilibrating slowly. (d) More than one kinetically distinct pool of VLDL triglyceride with precursors equilibrating slowly. 


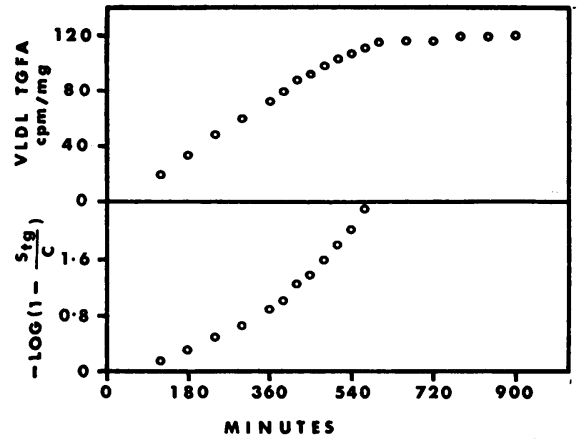

FIGURE 2 Subject 1, upper curve. Specific activity-time plot of VLDL TGFA during a constant infusion of palmitic acid- $1-{ }^{14} \mathrm{C}$. Lower curve, the VLDL TGFA specific activity expressed as $-\log \left(1-\left[\mathrm{S}_{\mathrm{TG}} / \mathrm{C}\right]\right)$ (see text) and plotted against time.

If isotopic equilibration of the immediate precursor of VLDL-TGFA is not instantaneous, $\mathrm{S}_{\mathrm{TG}}$ and hence the value of $-\log \left(1-\left[\mathrm{S}_{\mathrm{TG}} / \mathrm{C}\right]\right)$ at any given time will be lower than that computed from equation 1 . However, as the specific activity of the immediate precursor approaches $\mathrm{C}$, the gradient of the curve plotted by $-\log \left(1-\left[\mathrm{S}_{\mathrm{TG}} / \mathrm{C}\right]\right)$ against time will increase towards that of the theoretical curve until it achieves linearity with a gradient of $\mathrm{k}$ (Fig. 1c). Such a curve would also describe the situation in which TGFA transported in smaller VLDL are derived from larger VLDL. The TGFA in the larger VLDL could be regarded as the immediate precursor of that in the smaller VLDL. Consequently the slope of the curve of $-\log \left(1-\left[\mathrm{S}_{\mathrm{TG}} / \mathrm{C}\right]\right)$ against time for total VLDL-TGFA would continue to increase until the TGFA in all but the smallest VLDL have achieved isotopic equilibration with their respective immediate precursors (Fig. 1c).

Fig. $1 d$ shows a curve which may result from a combination of the situations producing curves $b$ and $c$; this curve will reflect whichever of the two situations

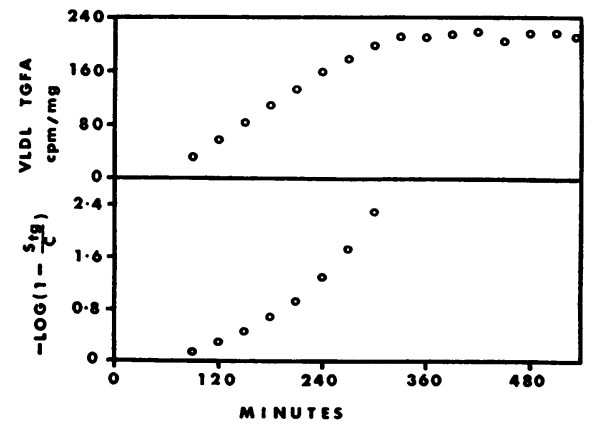

Figure 3 Subject 7, upper curve. Specific activity-time plot of VLDL TGFA during constant infusion of palmitic acid- $1-{ }^{14} \mathrm{C}$. Lower curve. Plot of $-\log \left(1-\left[\mathrm{S}_{\mathrm{TG}} / \mathrm{C}\right]\right)$ against time. predominates: i.e. it could show an increasing gradient, a decreasing gradient or could even be linear.

Any comparison between experimental curves and those shown in Fig. 1 can only be at a qualitative level. A decreasing slope will suggest the existance of independently metabolized pools of VLDL-TGFA possessing distinct (fractional) turnover rates, while an increasing slope suggest incomplete isotopic equilibration of precursors of VLDL-TGFA or the presence in the plasma of a precursor-product relationship between different components of VLDL-TGFA.

\section{RESULTS}

Constant infusions. Figs. 2 and 3 show the TGFA specific activity-time curves in total VLDL during constant infusions of palmitic acid-1- ${ }^{14} \mathrm{C}$ in two subjects. The results from all eight experiments are presented in Table I. There was a wide variation in the time required

TABLE I

Specific Activities of VLDL Triglyceride Fatty Acids during Constant Infusions of Palmitic Acid-1-14C

\begin{tabular}{|c|c|c|c|c|c|c|c|c|}
\hline \multirow[b]{2}{*}{$\begin{array}{c}\text { Subject. } \\
\text { VLDL TG } \\
\text { concen- } \\
\text { tration*. } \\
\text { Timeł }\end{array}$} & \multicolumn{8}{|c|}{ VLDL Triglyceride specific activity } \\
\hline & .270 & 130 & 108 & 160 & 59 & 73 & 89 & 63 \\
\hline & \multicolumn{8}{|c|}{$c p m / m g$} \\
\hline 90 & - & - & 28 & 51 & 44 & 33 & 31 & 68 \\
\hline 120 & 19 & 12 & 49 & 75 & 75 & 53 & 56 & 107 \\
\hline 150 & - & - & 70 & 103 & 98 & 67 & 82 & 136 \\
\hline 180 & 33 & 23 & 89 & 122 & 121 & 84 & 108 & 175 \\
\hline 210 & - & 30 & 111 & 138 & 133 & 96 & 132 & 202 \\
\hline 240 & 48 & 34 & 127 & 168 & 145 & 109 & 158 & 233 \\
\hline 270 & - & 39 & 140 & 183 & 176 & 117 & 178 & 247 \\
\hline 300 & 59 & 45 & 159 & 186 & 184 & 126 & 197 & 256 \\
\hline 330 & - & 50 & 174 & 203 & 203 & 137 & 212 & 260 \\
\hline 360 & 72 & 56 & 183 & 202 & 212 & 140 & 211 & 263 \\
\hline 390 & 79 & 58 & 188 & 207 & 227 & 150 & 216 & 256 \\
\hline 420 & 88 & 62 & 191 & 211 & 233 & 152 & 219 & 260 \\
\hline 450 & 92 & 63 & 195 & 208 & 234 & 154 & 205 & \\
\hline 480 & 98 & 67 & 199 & 210 & 236 & 149 & 218 & \\
\hline 510 & 103 & 70 & 200 & 208 & 236 & 152 & 218 & \\
\hline 540 & 107 & 76 & 200 & 212 & 230 & 153 & 211 & \\
\hline 570 & 111 & 79 & 198 & & & & & \\
\hline 600 & 115 & 84 & 202 & & & & & \\
\hline 660 & 120 & 83 & & & & & & \\
\hline 720 & 120 & 83 & & & & & & \\
\hline 780 & 123 & & & & & & & \\
\hline 840 & 123 & & & & & & & \\
\hline 900 & 124 & & & & & & & \\
\hline
\end{tabular}

* The VLDL triglyceride concentration is expressed in $\mathrm{mg} / 100 \mathrm{ml}$

$\ddagger$ Minutes after commencement of infusion. 
for complete isotopic equilibration of VLDL-TGFA (5-11 hr), being generally shorter when the TGFA pools were small. There was, however, a marked similarity in the pattern of the VLDL-TGFA specific activity $\left(\mathrm{S}_{\mathrm{TG}}\right)$-time curves in all studies. The curves are dominated by a long linear segment followed by an abrupt flattening to a constant level (Figs. 2 and 3). A more critical assessment of the curves can be made by plotting $-\log \left(1-\left[\mathrm{S}_{\mathrm{T} \sigma} /\right.\right.$ equilibrated $\left.\left.\mathrm{S}_{\mathrm{T} \mathrm{G}}\right]\right)$ against time (see theoretical considerations). The lower segments of Figs. 2 and 3 show two such plots from $90 \mathrm{~min}$ until $\mathrm{S}_{\text {тa }}$ had reached $90 \%$ of the equilibrated level. ${ }^{4}$ Table II presents the values from all studies. In every case the gradient of $-\log \left(1-\left[\mathrm{S}_{\mathrm{TG}} /\right.\right.$ equilibrated $\left.\left.\mathrm{S}_{\mathrm{TG}}\right]\right)$ against time increased progressively with time and could not in any single case be described in terms of a single exponential function. The existence of differing (fractional) turnover rates of TGFA in independently metabolized pools of VLDL would have resulted in a decreasing gradient; consequently there is no support for such a suggestion. The findings were consistent with

${ }^{4}$ As complete equilibration is approached the accuracy of values $1-\left[\mathrm{S}_{\mathrm{TG}}\right.$ /equilibrated $\left.\mathrm{S}_{\mathrm{TG}}\right]$ becomes increasingly suspect. We have not computed values beyond $90 \%$.
TABLE II

Values for $-\log \left(1-\left[S_{T G^{*}} /\right.\right.$ equilibrated $\left.\left.S_{T G}\right]\right) d u r i n g$ Constant Infusion of Palmitic Acid-1 $1{ }^{14} \mathrm{C}$

\begin{tabular}{|c|c|c|c|c|c|c|c|c|}
\hline \multirow[b]{2}{*}{$\begin{array}{c}\text { Subject. } \\
\text { Time }\end{array}$} & \multicolumn{8}{|c|}{$-\log \left(1-\left[\mathrm{S}_{\mathrm{TG}} /\right.\right.$ equilibrated $\left.\left.\mathrm{S}_{\mathrm{TG}}\right]\right)$} \\
\hline & $\ldots 1$ & 2 & 3 & 4 & 5 & 6 & 7 & 8 \\
\hline 90 & & & 0.15 & 0.28 & 0.21 & 0.24 & 0.15 & 0.30 \\
\hline 120 & 0.17 & 0.16 & 0.28 & 0.44 & 0.38 & 0.43 & 0.30 & 0.53 \\
\hline 150 & & & 0.43 & 0.68 & 0.54 & 0.58 & 0.47 & 0.74 \\
\hline 180 & 0.31 & 0.32 & 0.59 & 0.87 & 0.72 & 0.81 & 0.69 & 1.12 \\
\hline 210 & & 0.45 & 0.81 & 1.07 & 0.83 & 1.00 & 0.93 & 1.51 \\
\hline 240 & 0.49 & 0.52 & 1.01 & 1.61 & 0.96 & 1.26 & 1.29 & 2.27 \\
\hline 270 & & 0.63 & 1.20 & 2.06 & 1.38 & 1.47 & 1.70 & \\
\hline 300 & 0.65 & 0.78 & 1.58 & 2.18 & 1.52 & 1.77 & 2.35 & \\
\hline 330 & & 0.92 & 2.04 & & 1.99 & 2.31 & & \\
\hline 360 & 0.88 & 1.11 & 2.47 & & 2.31 & & & \\
\hline 390 & 1.02 & 1.19 & & & & & & \\
\hline 420 & 1.25 & 1.36 & & & & & & \\
\hline 450 & 1.37 & 1.41 & & & & & & \\
\hline 480 & 1.58 & 1.63 & & & & & & \\
\hline 510 & 1.80 & 1.83 & & & & & & \\
\hline 540 & 2.02 & 2.43 & & & & & & \\
\hline 570 & 2.30 & & & & & & & \\
\hline
\end{tabular}

* STa, specific activity of VLDL TGFA (cpm/mg).

$\ddagger$ Minutes after commencement of infusion.

slow isotopic equilibration of precursors of VLDLTGFA or with the presence in the plasma of a pre-

TABLE III

Specific Activities of Triglyceride Fatty Acids in Two Subfractions of VLDL during a Constant Infusion of Palmitic Acid-1 $-{ }^{14} \mathrm{C}$

\begin{tabular}{|c|c|c|c|c|c|c|}
\hline \multirow{3}{*}{$\begin{array}{c}\text { Subject..... } \\
\text { Triglyceride } \\
\text { concentration*. } \\
\text { Time }\end{array}$} & \multicolumn{6}{|c|}{ Triglyceride fatty acid specific activity } \\
\hline & \multicolumn{2}{|c|}{9} & \multicolumn{2}{|c|}{10} & \multicolumn{2}{|c|}{11} \\
\hline & $\begin{array}{l}\text { Sf }>100 \\
\ldots 20\end{array}$ & $\begin{array}{c}\text { Sf } 20-100 \\
21\end{array}$ & $\begin{array}{c}\text { Sf }>100 \\
19\end{array}$ & $\begin{array}{c}\text { Sf } 20-100 \\
29\end{array}$ & $\begin{array}{c}\text { Sf }>100 \\
57\end{array}$ & $\begin{array}{c}\text { Sf } 20-100 \\
42\end{array}$ \\
\hline & \multicolumn{6}{|c|}{$c p m / m g$} \\
\hline 60 & 93 & 57 & 48 & 29 & & \\
\hline 90 & 169 & 118 & 83 & 71 & & \\
\hline 120 & 221 & 185 & 161 & 122 & 244 & 185 \\
\hline 150 & 264 & 235 & 210 & 203 & 344 & 256 \\
\hline 180 & 307 & 279 & 265 & 243 & 385 & 358 \\
\hline 210 & 365 & 336 & 350 & 310 & 453 & 456 \\
\hline 240 & 411 & 393 & 378 & 353 & 489 & 453 \\
\hline 270 & 402 & 433 & 425 & 388 & 622 & 580 \\
\hline 300 & 440 & 426 & 465 & 425 & 656 & 610 \\
\hline 330 & 432 & 462 & 432 & 454 & 717 & 639 \\
\hline 360 & 438 & 448 & 485 & 460 & 782 & 769 \\
\hline 390 & & & 476 & 480 & 808 & 709 \\
\hline 420 & & & & & 821 & 792 \\
\hline 450 & & & & & 855 & 754 \\
\hline 480 & & & & & 781 & 829 \\
\hline 510 & & & & & 846 & 821 \\
\hline 540 & & & & & 805 & 857 \\
\hline
\end{tabular}

* Triglyceride concentrations are expressed as $\mathrm{mg} / 100 \mathrm{ml}$.

$\ddagger$ Minutes after commencement of palmitic acid-1-14 $\mathrm{C}$ infusion. 


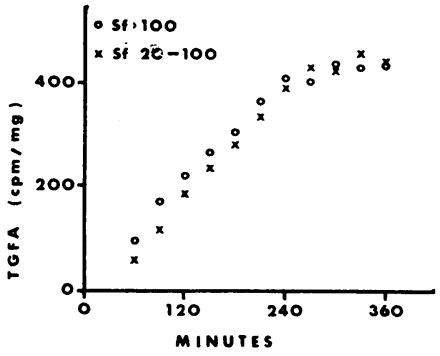

FIGURE 4 Subject 9, specific activity-time plots for TGFA in two subclasses of VLDL ( $\mathrm{Sf}>100$ and $\mathrm{Sf} 20-100)$ during a constant infusion of palmitic acid- $1-{ }^{14} \mathrm{C}$.

cursor-product relationship between different components of VLDL-TGFA. Because none of the curves of $-\log \left(\left[\mathrm{S}_{\mathrm{TG}} /\right.\right.$ equilibrated $\left.\left.\mathrm{S}_{\mathrm{TG}}\right]\right)$ against time became linear it was not possible to estimate the (fractional) turnover rates.

TGFA specific activities were also measured in two VLDL subfractions during constant infusions of palmitic acid- $1{ }^{14} \mathrm{C}$ in subjects $9-11$ (Table III). The specific activity of TGFA in the $\mathrm{Sf}>100$ fraction was higher at each time point than in the Sf 20-100 fraction until equilibration was complete (Fig. 4). After the first hour of infusion and until shortly before isotopic equilibration

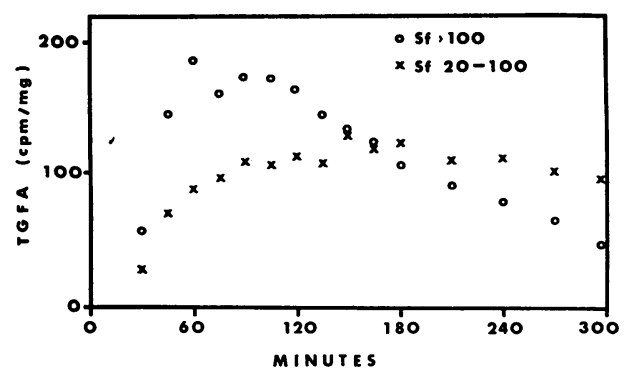

FigURE 5 Subject 13, specific activity-time plots for TGFA in two subclasses of VLDL ( $\mathrm{Sf}>100$ and $\mathrm{Sf} 20-100$ ) after a single intravenous injection of palmitic acid-1- ${ }^{14} \mathrm{C}$.

was complete, the two curves in all three subjects were approximately parallel. There was therefore no suggestion that the TGFA in the two subfractions had differing (fractional) turnover rates. The results would, however, be consistent with a precursor-product relationship between TGFA in larger and smaller VLDL.

Single injection. The TGFA specific activities in two subfractions of VLDL ( $\mathrm{Sf}>100$ and $\mathrm{Sf} 20-100$ ) were also measured after a single intravenous injection of palmitic acid- $1-{ }^{14} \mathrm{C}$. One such experiment is shown in Fig. 5 and Table IV presents the findings in the four subjects 12-15. There was a striking and consistent

TABLE IV

Specific Activities of Triglyceride Fatty Acids in Subclasses of VLDL after a Single Injection of Palmitic Acid-1 $1{ }^{14} \mathrm{C}$

\begin{tabular}{|c|c|c|c|c|c|c|c|c|}
\hline \multirow{3}{*}{$\begin{array}{l}\text { Subject....... } \\
\text { Triglyceride } \\
\text { concentration*.. } \\
\text { Time }\end{array}$} & \multicolumn{8}{|c|}{ Triglyceride fatty acid specific activity } \\
\hline & \multicolumn{2}{|c|}{12} & \multicolumn{2}{|c|}{13} & \multicolumn{2}{|c|}{14} & \multicolumn{2}{|c|}{15} \\
\hline & $\begin{array}{l}\text { Sf }>100 \\
\ldots .358\end{array}$ & $\begin{array}{c}\text { Sf } 20-100 \\
155\end{array}$ & $\begin{array}{c}\text { Sf }>100 \\
89\end{array}$ & $\begin{array}{c}\text { Sf } 20-100 \\
51\end{array}$ & $\begin{array}{c}\text { Sf }>100 \\
54\end{array}$ & $\begin{array}{c}\text { Sf } 20-100 \\
69\end{array}$ & $\begin{array}{c}\text { Sf }>100 \\
17\end{array}$ & $\begin{array}{c}\text { Sf } 20-100 \\
23\end{array}$ \\
\hline & \multicolumn{8}{|c|}{$c p m / m g$} \\
\hline 30 & 23 & 16 & 58 & 28 & 29 & 24 & 161 & 103 \\
\hline 45 & 51 & 31 & 143 & 71 & 65 & 56 & 383 & 242 \\
\hline 60 & 72 & 38 & 187 & 88 & 90 & 70 & 443 & 329 \\
\hline 75 & 81 & 49 & 160 & 96 & 98 & 82 & 449 & 421 \\
\hline 90 & 93 & 53 & 174 & 108 & 114 & 84 & 562 & 473 \\
\hline 105 & 76 & 60 & 171 & 106 & 128 & 105 & 487 & 431 \\
\hline 120 & 90 & 62 & 165 & 113 & 117 & 109 & 393 & 432 \\
\hline 135 & 86 & 57 & 145 & 108 & 110 & 112 & 342 & 408 \\
\hline 150 & 83 & 61 & 134 & 130 & 107 & 97 & 328 & 391 \\
\hline 165 & 84 & 60 & 125 & 119 & - & - & - & - \\
\hline 180 & 74 & 62 & 106 & 122 & 92 & 101 & 254 & 317 \\
\hline 210 & 68 & 58 & 91 & 110 & 82 & 99 & 198 & 323 \\
\hline 240 & 63 & 60 & 77 & 111 & 76 & 102 & 153 & 221 \\
\hline 270 & 59 & 62 & 65 & 101 & 64 & 82 & 121 & 211 \\
\hline 300 & 53 & 61 & 48 & 97 & - & - & 94 & 168 \\
\hline 330 & 55 & 62 & 46 & 78 & - & - & 83 & 142 \\
\hline
\end{tabular}

* Triglyceride concentrations are expressed as $\mathrm{mg} / 100 \mathrm{ml}$. $\ddagger$ Minutes after injection of palmitic acid-1-14 $\mathrm{C}$. 
difference between the two subfractions in all studies. The TGFA specific activity in Sf $>100$ VLDL rose more quickly and reached a higher peak sooner than TGFA in the Sf $20-100$ fraction. The specific-activity time curves of the two subfractions crossed in a manner that further suggested a precursor-product relationship. These curves, however, were also consistent with differences in the (fractional) turnover rates of independently metabolized pools of TGFA in VLDL of different sizes.

\section{DISCUSSION}

Constant infusions. Considering the studies with total VLDL first, the results were compatible with slow isotopic equilibration anywhere between plasma FFA and TGFA in the final smallest species of VLDL, assuming that progressive conversion of larger to smaller lipoproteins does occur. There was no evidence for independently metabolized pools of TGFA possessing differing (fractional) turnover rates.

The studies in which two subfractions of VLDL (Sf $>100$ and Sf 20-100) were analyzed separately also failed to show that TGFA in VLDL of different sizes are independently metabolized at different (fractional) turnover rates. Rather, the results were consistent with TGFA in the larger VLDL being a precursor of that in the smaller VLDL. Delayed isotopic equilibration of precursors of VLDL-TGFA, a possibility suggested with studies of total VLDL, could not alone account for the differences between the two subfractions.

Single injections. These studies resulted in specific activity-time curves that were compatible with classical precursor-product relationships between TGFA in Sf $>$ 100 and Sf 20-100 VLDL, although the possibility could not be excluded that the TGFA in the two subfractions represented two independently metabolized pools.

In summary, the results of all experiments when considered together, are most readily compatible with the concept of a precursor-product relationship between TGFA in larger and smaller VLDL.

The possible conversion of very low density lipoproteins to lipoproteins of higher density has been strongly implied in several studies $(5,13-15)$. A precursor-product relationship between the pools of TGFA transported in VLDL and low density lipoproteins has been suggested on the basis of specific activity-time curves for TGFA transported in the two lipoprotein classes after an injection of palmitic acid-1 $-{ }^{14} \mathrm{C}(1,16)$. In this study we have found a similar relationship between TGFA transported in larger and smaller VLDL (Fig. 5). All our experiments are compatible with the suggestion that a proportion of the TGFA transported in smaller VLDL had initially been secreted into the plasma in larger ones. Two possibilities exist for such a precursor-product relationship between the TGFA in larger and smaller VLDL. There could be a direct transfer of TGFA molecules from larger to smaller VLDL, or perhaps more likely on the basis of findings in vitro (15), larger VLDL become progressively smaller as triglyceride is removed. The formation of LDL may represent the final step in this process.

While stepwise degradation of lipoproteins has been clearly shown in vitro (15), some published in vivo studies $(5,13,14)$ could also be interpreted to show that TGFA in larger lipoproteins are cleared more efficiently than smaller species. When lipoproteins of different size have been injected intravenously in humans, TGFA transported in chylomicrons have been found to have a higher (fractional) turnover rate than those in VLDL (17-19). Furthermore, Quarfordt and Goodman (20), who injected different species of chylomicrons into rats, reported that the rate at which chylomicron TGFA was cleared was proportional to particle size. However, it is possible that reinjected lipoproteins do not represent accurately the turnover of endogenously produced species, possibly because of partial sequestration in the liver (21). Certainly an analysis of the specific-activity-time curves for TGFA in total VLDL (Figs. 2 and 3) and in subfractions of VLDL (Fig. 4) during constant infusions of radiopalmitate gave no indication of the existence of independently metabolized pools of VLDL-TGFA with differing (fractional) turnover rates. Although our results therefore do not support the concept that the rate at which TGFA is removed from plasma is a function of VLDL size, this cannot be altogether excluded since the isotope kinetics might have been dominated by the precursor-product relationship between components of VLDL-TGFA to an extent that masked all other influences.

Finally, it should be emphasized that regardless of the mechanism, the observed differences found between the VLDL subfractions clearly show that in terms of isotope kinetics the VLDL-TGFA cannot be described as a single pool.

\section{ACKNOWLEDGMENTS}

We should like to thank Mrs. Geraldine Power for her technical assistance.

This work was supported in part by a grant from the National Heart Foundation of Australia.

\section{REFERENCES}

1. Havel, R. J. 1961. Conversion of plasma free fatty acids into triglycerides of plasma lipoproteins in man. Metab. (Clin. Exp.). 10: 1031.

2. Lossow, W. J., F. T. Lindgren, J. C. Murchio, G. R. Stevens, and L. C. Jensen. 1969. Particle size and protein content of six fractions of the Sf $>20$ plasma 
lipoprotein isolated by density gradient centrifugation. J. Lipid Res. 10: 68.

3. Schonfeld, G. 1970. Changes in the composition of very low density lipoproteins during carbohydrate induction in man. J. Lab. Clin. Med. 75: 206.

4. Gustafson, A., P. Alaupovic, and R. H. Tiernan. 1965. Studies of the composition and structure of serum lipoproteins: isolation, purification and characterization of VLDL of human serum. Biochemistry. 4: 596.

5. Barter, P. J., and P. J. Nestel. 1970. The distribution of triglyceride in subclasses of very low density plasma lipoproteins. J. Lab. Clin. Med. 76: 925.

6. Fredrickson, D. S., R. I. Levy, and R. S. Lees. 1967. Fat transport in lipoproteins-An integrated approach to mechanisms and disorders. N. Engl. J. Med. 276: $32,94,148,215$, and 273.

7. Fredrickson, D. S., and R. S. Gordon, Jr. 1958. The metabolism of albumin bound ${ }^{14} \mathrm{C}$-labeled unesterified fatty acids in normal human subjects. J. Clin. Invest. 37: 1504 .

8. Havel, R. J., H. A. Eder, and J. H. Bragdon. 1955. Distribution and chemical composition of ultracentrifugally separated lipoproteins in human serum. J. Clin. Invest. $34: 1345$.

9. Dole, V. P. 1956. A relation between non-esterified fatty acids in plasma and the metabolism of glucose. J. Clin. Invest. $35: 150$.

10. Lloyd, M. R., and R. B. Goldrick. 1968. A simplified method for estimating plasma triglyceride: their stability during cold storage. Med. J. Aust. 2: 493.

11. Borgström, B. 1959. Investigation of lipid separation methods. Separation of cholesterol esters, glycerides and free fatty acids. Acta Physiol. Scand. 25: 111.
12. Zilversmit, D. B. 1960 . The design and analysis of isotope experiments. Amer. J. Med. 29: 832.

13. Lindgren, F. T., N. K. Freeman, A. V. Nichols, and J. W. Gofman. 1956. The blood lipids and the clearing factor. Int. Conf. Biochem. Probl. Lipids 3rd, 1956. 224.

14. Lindgren, F. T., and A. V. Nichols. 1960. Structure and function of human serum lipoproteins. Plasma Protein. $2: 1$.

15. Shore, B., and V. Shore. 1962. Some physical and chemical properties of the lipoproteins produced by lipolysis of human serum Sf 20-400 lipoprotein by postheparin plasma. J. Atheroscler. Res. 2: 104.

16. Quarfordt, S. H., A Frank, D. M. Shames, M. Berman, and D. Steinberg. 1970. Very low density lipoprotein triglyceride transport in type IV hyperlipoproteinemia and the effects of carbohydrate-rich diets. J. Clin. Invest. 49 : 2281.

17. Nestel, P. J. 1964. Relationship between plasma triglyceride and removal of chylomicrons. J. Clin. Invest. 43: 943.

18. Farquhar, J. W., R. C. Gross, R. M. Wagner, and G. M. Reaven. 1965. Validation of an incompletely coupled two-compartment non-recycling catenary model for turnover of liver and plasma triglycerides in man. J. Lipid Res. 6: 119.

19. Eaton, R. P., M. Berman, and D. Steinberg. 1969. Kinetics of plasma free fatty acid and triglyceride metabolism in man. J. Clin. Invest. 48: 1560.

20. Quarfordt, S. H., and D. S. Goodman. 1966. Heterogeneity in rate of plasma clearance of chylomicrons of different sizes. Biochim. Biophys. Acta. 116: 382.

21. Felts, J. M. 1965. The metabolism of chylomicron triglyceride fatty acids by perfused rat livers and by intact rats. Ann. N. Y. Acad. Sci. 131: 24. 